\title{
Topological Structures of Derivative Weighted Composition Operators on the Bergman Space
}

\author{
Ce-Zhong Tong, ${ }^{1}$ Cheng Yuan, ${ }^{2}$ and Ze-Hua Zhou ${ }^{3,4}$ \\ ${ }^{1}$ Department of Mathematics, Hebei University of Technology, Tianjin 300401, China \\ ${ }^{2}$ Institute of Mathematics, School of Science, Tianjin University of Technology and Education, Tianjin 300222, China \\ ${ }^{3}$ Department of Mathematics, Tianjin University, Tianjin 300072, China \\ ${ }^{4}$ Center for Applied Mathematics, Tianjin University, Tianjin 300072, China
}

Correspondence should be addressed to Ze-Hua Zhou; zehuazhoumath@aliyun.com

Received 24 July 2015; Accepted 20 October 2015

Academic Editor: Nikolai L. Vasilevski

Copyright (C) 2015 Ce-Zhong Tong et al. This is an open access article distributed under the Creative Commons Attribution License, which permits unrestricted use, distribution, and reproduction in any medium, provided the original work is properly cited.

We characterize the difference of derivative weighted composition operators on the Bergman space in the unit disk and determine when linear-fractional derivative weighted composition operators belong to the same component of the space of derivative weighted composition operators on the Bergman space under the operator norm topology.

\section{Introduction}

Let $\mathbb{D}$ be the unit disk in the complex plane. The algebra of all holomorphic functions on domain $\mathbb{D}$ will be denoted by $H(\mathbb{D})$. Let $S(\mathbb{D})$ be the set of analytic self-maps of $\mathbb{D}$. Every $\varphi \in S(\mathbb{D})$ induces the composition operator $C_{\varphi}$ defined by $C_{\varphi} f=f \circ \varphi$ for $f \in H(\mathbb{D})$.

Let $u: \mathbb{D} \rightarrow \mathbb{C}$ be analytic, and the weighted composition operator, $u C_{\varphi}$, is defined by

$$
u C_{\varphi}(f)(z)=u(z) \cdot f(\varphi(z))
$$

for any $f \in H(\mathbb{D})$ and $z \in \mathbb{D}$. When $u \equiv 1$, it becomes a composition operator.

Derivative weighted composition ( $d$-composition) operator is defined by

$$
D_{\varphi}(f)=\varphi^{\prime} f \circ \varphi .
$$

It is obvious that $D_{\varphi}=\varphi^{\prime} C_{\varphi}$.

For $\alpha>-1$ and $0<p<\infty$, we recall that a weighted Bergman space $A_{\alpha}^{p}(\mathbb{D})$ is the set of holomorphic functions on the unit disk for which

$$
\int_{\mathbb{D}}|f(z)|^{p}\left(1-|z|^{2}\right)^{\alpha} \frac{d A(z)}{\pi}<\infty,
$$

where $d A(z)$ is the Lebesgue area measure on the unit disk and $\|f\|_{p}$ is the $p$ th root of this integral. Moreover, the Bergman space $A^{2}(\mathbb{D})=A_{0}^{2}(\mathbb{D})$ is a Hilbert space with the inner product

$$
\langle f, g\rangle=\int_{\mathbb{D}} f(z) \overline{g(z)} \frac{d A(z)}{\pi} .
$$

A weighted Dirichlet space $\mathscr{D}_{\alpha}^{p}$ is the set of holomorphic functions on the unit disk for which

$$
\int_{\mathbb{D}}\left|f^{\prime}(z)\right|^{p}\left(1-|z|^{2}\right)^{\alpha} \frac{d A(z)}{\pi}<\infty
$$

with norm given by

$$
\|f\|_{\mathscr{D}_{\alpha}}^{p}=|f(0)|^{p}+\int_{\mathbb{D}}\left|f^{\prime}(z)\right|^{p}\left(1-|z|^{2}\right)^{\alpha} \frac{d A(z)}{\pi}
$$

and the Dirichlet space $\mathscr{D}=\mathscr{D}_{0}^{2}$ is also a Hilbert space with the inner product

$$
\langle f, g\rangle=f(0) \overline{g(0)}+\int_{\mathbb{D}} f^{\prime}(z) \overline{g^{\prime}(z)} \frac{d A(z)}{\pi} .
$$

It is easy to show that (Exercise 2.1.6 in [1]) $A_{\alpha}^{2}(\mathbb{D})=\mathscr{D}_{\alpha+2}$. 
Much effort has been expended on characterizing those analytic maps which induce bounded or compact composition operators. Readers interested in this topic can refer to the books [2] by Shapiro, [1] by Cowen and MacCluer, and $[3,4]$ by Zhu, which are excellent sources for the development of the theory of composition operators and function spaces, and the recent papers [5-8].

Another area of particular interest is the topological structure of the space of composition operators acting on a given function space. When $X$ is a Banach space of analytic functions, we write $C(X)$ for the space of composition operators and $C_{d}(X)$ for the space of derivative weighted composition operators on $X$ under the operator norm topology. The investigation of the topological structure of $C\left(H^{2}\right)$ was initiated by Berkson [9] in 1981. Central problem focuses on the relations between the structure of $C\left(H^{2}\right)$ and the compactness properties of its members.

Continuing the work, in 1989, MacCluer [10] showed that, on the weighted Bergman space $A_{\alpha}^{2}$ for $\alpha \geq-1$, the compact composition operators form an arcwise connected set in $C\left(A_{\alpha}^{2}\right)$ and gave necessary conditions for two composition operators to have compact difference. At about the same time, Shapiro and Sundberg [11] gave further results on compact difference and isolation and, among other things, posed the following fundamental question and conjectured that it had a positive answer:

(*) Do the compact composition operators form a connected component of the set $C\left(H^{2}\right)$ ?

In 2008, Gallardo-Gutiérrez et al. [12] gave a negative answer to the question for a variety of spaces in addition to $H^{p}$. In 2003, Bourdon [13] determined two linear-fractional self-maps of the disk having the same first-order data at a point $\xi$ on the boundary of the disk and different second derivatives at $\xi$ lie in the same component of $C\left(H^{2}\right)$, while the induced composition operators do not have compact difference. In 2005, Moorhouse [14] answers the question of compact difference for composition operators acting on $A_{\alpha}^{2}$, $\alpha>-1$, and gave a partial answer to the component structure of $C\left(A_{\alpha}^{2}\right)$. Later, Kriete and Moorhouse [15] extended their study to general linear combinations. Saukko $[16,17]$ obtained a complete characterization of bounded and compact differences between standard weighted Bergman spaces. Recently, Choe et al. $[18,19]$ extend Moorhouses characterization to the unit polydisk and unit ball in $\mathbb{C}^{N}$.

Topological structure of composition operators, which are from some analytic function spaces into the Bloch type spaces or the space of bounded analytic functions, has been studied intensively during the past decades. Interested readers can refer to $[20,21]$ and the references therein.

In 2004 Čučkovič and Zhao [5] characterized the bounded, compact, and Schatten class weighted composition operators on the Bergman space by generalized Berezin transforms. Derivative weighted composition operators are a special class of the weighted composition operators. The boundedness is characterized as follows.
Theorem 1 (Theorem 1 in [5]). Let $\varphi$ be an analytic self-map of $\mathbb{D}$. Then the d-weighted composition operator $D_{\varphi}$ is bounded on $A^{2}$ if and only if

$$
\int_{\mathbb{D}} \frac{\left(1-|z|^{2}\right)^{2}\left|\varphi^{\prime}(w)\right|^{2}}{|1-\bar{z} \varphi(w)|^{4}} d A(w)<\infty .
$$

Building on those foundations, the present paper continues this line of research. The remainder is assembled as follows. In Section 2, we characterize the compact difference of two derivative weighted composition operators on the Bergman space in the unit disk and discuss the isolation and component structure of the derivative weighted composition operator in $\mathscr{C}_{d}\left(A^{2}\right)$, and some similar results about composition operators on the Dirichlet space are also presented there. In Section 3, we show that the $d$-composition operators form an arcwise connected set in $\mathscr{C}_{d}\left(A^{2}\right)$. Finally, we determine when linear-fractional $d$-composition operators belong to the same component on the Bergman space under the operator norm topology in Section 4.

\section{Difference of Derivative Weighted Composition Operators}

Our discussion begins with the characterization of the compact difference of $d$-composition operators. When we accomplished the following theorem, we read a new paper [22], in which Allen et al. obtained the same estimation (Lemma 10). Nonetheless, we present our version in the following, since our method is totally different.

Theorem 2. Suppose $\varphi: \mathbb{D} \rightarrow \mathbb{D}$ has finite angular derivative $\varphi^{\prime}(\zeta)$ at some point $\zeta$ in the unit circle. Let $\psi: \mathbb{D} \rightarrow \mathbb{D}$ and consider the difference of $D_{\varphi}$ and $D_{\psi}$ acting on the Bergman space $A^{2}(\mathbb{D})$. Then we have $\left\|D_{\varphi}-D_{\psi}\right\|_{e}^{2} \geq 1$ if the symbol $\psi$ satisfies one of the following two conditions:

(i) $\psi(\zeta) \neq \varphi(\zeta)$ (as radial limits).

(ii) $\psi(\zeta)=\varphi(\zeta)$ but $\psi^{\prime}(\zeta) \neq \varphi^{\prime}(\zeta)$.

Proof. Since the Bergman space $A^{2}(\mathbb{D})$ is rotationally invariant, we may assume $\zeta=1, \varphi(1)=1$, and $\varphi^{\prime}(1)=s<\infty$. We first estimate $\left\|D_{\varphi}-D_{\psi}\right\|$ by considering the adjoint of $D_{\varphi}-D_{\psi},\left(D_{\varphi}-D_{\psi}\right)^{*}$ acting on reproducing kernel functions $K_{z}(w)=(1-\bar{z} w)^{-2}$. Since

$$
\begin{aligned}
& \left\|\left(D_{\varphi}-D_{\psi}\right)^{*} K_{z}\right\|^{2} \\
& =\left\langle\left(D_{\varphi}-D_{\psi}\right)^{*} K_{z},\left(D_{\varphi}-D_{\psi}\right)^{*} K_{z}\right\rangle \\
& =\left\langle\overline{\varphi^{\prime}(z)} K_{\varphi(z)}-\overline{\psi^{\prime}(z)} K_{\psi(z)} \overline{\varphi^{\prime}(z)} K_{\varphi(z)}\right. \\
& \left.-\overline{\psi^{\prime}(z)} K_{\psi(z)}\right\rangle=\left|\varphi^{\prime}(z)\right|^{2}\left\|K_{\varphi(z)}\right\|^{2}+\left|\psi^{\prime}(z)\right|^{2} \\
& \cdot\left\|K_{\psi(z)}\right\|^{2}-2 \operatorname{Re}\left\{\overline{\varphi^{\prime}(z)} \psi^{\prime}(z) K_{\varphi(z)}(\psi(z))\right\},
\end{aligned}
$$


the formulas for the kernel functions $K_{z}$ and their norms show that

$$
\begin{aligned}
& \left\|D_{\varphi}-D_{\psi}\right\|^{2} \geq \frac{\left\|\left(D_{\varphi}-D_{\psi}\right)^{*} K_{z}\right\|^{2}}{\left\|K_{z}\right\|^{2}} \\
& =\left|\varphi^{\prime}(z)\right|^{2}\left(\frac{1-|z|^{2}}{1-|\varphi(z)|^{2}}\right)^{2} \\
& +\left|\psi^{\prime}(z)\right|^{2}\left(\frac{1-|z|^{2}}{1-|\psi(z)|^{2}}\right)^{2} \\
& \quad-2 \operatorname{Re}\left\{\frac{1-|z|^{2}}{\varphi^{\prime}(z)} \psi^{\prime}(z)\left(\frac{\overline{\varphi(z)} \psi(z)}{1-\bar{\varphi}}\right)^{2}\right\}
\end{aligned}
$$

for all $z$ in $\mathbb{D}$.

By the Julia-Carathéodory Theorem, $\left(1-|z|^{2}\right) /\left(1-|\varphi(z)|^{2}\right)$ has nontangential limit $1 / s$ as $z$ goes to 1 , so the first term of the right-hand side in the above expression has nontangential limit 1 as $z$ goes to 1 .

To deal with the third term of the right-hand side in (10) we distinguish two cases.

Case 1. Consider $\lim _{r \rightarrow 1} \psi(r) \neq 1$. We can find $z_{n}$ approaching 1 nontangentially so that $\lim _{n \rightarrow \infty} \psi\left(z_{n}\right)=u \neq 1$.

From Schwarz-Pick Theorem we have that $\left|\psi^{\prime}\left(z_{n}\right)\right| \leq(1-$ $\left.\left|\psi\left(z_{n}\right)\right|^{2}\right) /\left(1-\left|z_{n}\right|^{2}\right)$ for every $n=1,2, \ldots$.

It follows that

$$
\begin{aligned}
& 2 \operatorname{Re}\left\{\overline{\varphi^{\prime}\left(z_{n}\right)} \psi^{\prime}\left(z_{n}\right)\left(\frac{1-\left|z_{n}\right|^{2}}{1-\overline{\varphi\left(z_{n}\right)} \psi\left(z_{n}\right)}\right)^{2}\right\} \\
& \quad \leq 2\left|\varphi^{\prime}\left(z_{n}\right)\right|\left|\psi^{\prime}\left(z_{n}\right)\right|\left|\frac{1-\left|z_{n}\right|^{2}}{1-\overline{\varphi\left(z_{n}\right)} \psi\left(z_{n}\right)}\right|^{2} \\
& \quad \leq \frac{2\left|\varphi^{\prime}\left(z_{n}\right)\right|}{\left|1-\overline{\varphi\left(z_{n}\right)} \psi\left(z_{n}\right)\right|^{2}}\left(1-\left|\psi\left(z_{n}\right)\right|^{2}\right)\left(1-\left|z_{n}\right|^{2}\right)
\end{aligned}
$$

has limit 0 as $n$ tends to infinity since

$$
\begin{aligned}
\lim _{n \rightarrow \infty}\left|\varphi^{\prime}\left(z_{n}\right)\right| & =s, \\
\lim _{n \rightarrow \infty}\left|1-\overline{\varphi\left(z_{n}\right)} \psi\left(z_{n}\right)\right| & =|1-u|, \\
\lim _{n \rightarrow \infty}\left(1-\left|z_{n}\right|^{2}\right) & =0 .
\end{aligned}
$$

Combining with the above discussions and by (10), we can conclude that $\left\|D_{\varphi}-D_{\psi}\right\|^{2} \geq 1$.

Case 2. Consider $\psi(1)=1$ but $\psi^{\prime}(1) \neq \varphi^{\prime}(1)=s$. We first consider

$$
\begin{aligned}
& \psi^{\prime}(z)^{-1}\left[\frac{1-\overline{\varphi(z)} \psi(z)}{1-|z|^{2}}\right]^{2}=\psi^{\prime}(z)^{-1}\left[\frac{1-|\varphi(z)|^{2}}{1-|z|^{2}}\right. \\
& \left.+\overline{\varphi(z)} \frac{\varphi(z)-\psi(z)}{1-|z|^{2}}\right]^{2}=\left[\psi^{\prime}(z)^{-1 / 2} \frac{1-|\varphi(z)|^{2}}{1-|z|^{2}}\right. \\
& +\overline{\varphi(z)} \frac{1-z}{1-|z|^{2}}\left(\psi^{\prime}(z)^{-1 / 2} \frac{1-\psi(z)}{1-z}\right. \\
& \left.\left.-\psi^{\prime}(z)^{-1 / 2} \frac{1-\varphi(z)}{1-z}\right)\right]^{2}
\end{aligned}
$$

Let $\gamma_{M}=\left\{z \in \mathbb{D}:|1-z| /\left(1-|z|^{2}\right)=M\right\}$, the boundary of a nontangential approach region at 1 . As $z$ approaches 1 along $\gamma_{M}$, the Julia-Carathéodory Theorem shows that

$$
\begin{gathered}
\frac{1-|\varphi(z)|^{2}}{1-|z|^{2}} \longrightarrow s, \\
\frac{1-\varphi(z)}{1-z} \longrightarrow s
\end{gathered}
$$

and either

$$
\frac{1-\psi(z)}{1-z} \longrightarrow \psi^{\prime}(1) \quad \text { when } \psi^{\prime}(1)<\infty
$$

or

$$
\left|\frac{1-\psi(z)}{1-z}\right| \rightarrow \infty \quad \text { when }\left|\psi^{\prime}(1)\right|=\infty
$$

It is easy to show that $\psi^{\prime}(z)^{-1 / 2}((1-\psi(z)) /(1-z))-$ $\psi^{\prime}(z)^{-1 / 2}((1-\varphi(z)) /(1-z)) \nrightarrow 0$. For any given positive number $N$, we may, by choosing $M$ large enough, find a sequence $z_{n}$ approaching 1 along $\gamma_{M}$ so that for $n$ large enough

$$
\begin{aligned}
& \mid \overline{\varphi\left(z_{n}\right)} \frac{1-z_{n}}{1-\left|z_{n}\right|^{2}} \psi^{\prime}\left(z_{n}\right)^{-1 / 2} \\
& \quad \cdot\left(\frac{1-\psi\left(z_{n}\right)}{1-z_{n}}-\frac{1-\varphi\left(z_{n}\right)}{1-z_{n}}\right) \mid>N+1 .
\end{aligned}
$$

It follows from (13) that

$$
\psi^{\prime}\left(z_{n}\right)^{-1}\left[\frac{1-\overline{\varphi\left(z_{n}\right)} \psi\left(z_{n}\right)}{1-\left|z_{n}\right|^{2}}\right]^{2}>N+1
$$

for $n$ large enough. So, for arbitrary positive $\delta$, choose $N$ such that $2 s / N<\delta$; we have

$$
\begin{aligned}
& 2 \operatorname{Re}\left\{\overline{\varphi^{\prime}\left(z_{n}\right)} \psi^{\prime}\left(z_{n}\right)\left(\frac{1-\left|z_{n}\right|^{2}}{1-\overline{\varphi\left(z_{n}\right)} \psi\left(z_{n}\right)}\right)^{2}\right\} \leq \frac{2 s}{N} \\
& \quad<\delta
\end{aligned}
$$


from which and (10), we deduce that $\left\|D_{\varphi}-D_{\psi}\right\|^{2} \geq 1-$ $\delta$ for arbitrary positive $\delta$, so $\left\|D_{\varphi}-D_{\psi}\right\|^{2} \geq 1$ by letting $\delta \rightarrow 0$.

It is standard to check the lower bound of the essential norm $\left\|D_{\varphi}-D_{\psi}\right\|_{e}^{2}$ :

$$
\begin{aligned}
\left\|D_{\varphi}-D_{\psi}\right\|_{e}^{2} & =\inf \left\{\left\|D_{\varphi}-D_{\psi}-Q\right\|^{2}: Q \text { compact }\right\} \\
& \geq \lim _{|z| \rightarrow 1}\left\|\left(D_{\varphi}-D_{\psi}\right)^{*}\left(\frac{K_{z}}{\left\|K_{z}\right\|}\right)\right\| \geq 1 .
\end{aligned}
$$

This completes the proof of the theorem.

As a direct consequence, we can obtain the following corollary.

Corollary 3. If $\varphi$ has finite angular derivative on a set of positive measures, then $D_{\varphi}$ is isolated in $\mathscr{C}_{d}\left(A^{2}\right)$. For any $\psi \neq$ $\varphi,\left\|D_{\varphi}-D_{\psi}\right\|_{e}^{2} \geq 1$.

Definition 4. Let $\varphi$ and $\psi$ be two self-mappings of the unit disk, and we say that $\varphi$ and $\psi$ have the same first-order boundary data or satisfy SBD, provided that whenever either $|\varphi(\zeta)|=1$ or $|\psi(\zeta)|=1$, then $\varphi(\zeta)=\psi(\zeta)$ and $\varphi^{\prime}(\zeta)=$ $\psi^{\prime}(\zeta)$

Theorem 5. Suppose $D_{\psi}$ is in the component of $\mathscr{C}_{d}\left(A^{2}\right)$ containing $D_{\varphi}$. Then $\varphi$ and $\psi$ must have the same first-order boundary data, where $\varphi$ has finite angular derivative.

Proof. Suppose either $|\varphi(\zeta)|=1$ or $|\psi(\zeta)|=1$ at $\zeta$ in the unit circle. If $\psi(\zeta) \neq \varphi(\zeta)$ or $\psi(\zeta)=\varphi(\zeta)$ but $\varphi^{\prime}(\zeta) \neq \psi^{\prime}(\zeta)$, by Theorem 2, $\left\|D_{\varphi}-D_{\psi}\right\|_{e}^{2} \geq 1, D_{\psi}$ is not in the component of $\mathscr{C}_{d}\left(A^{2}\right)$ containing $D_{\varphi}$. Hence $\psi(\zeta)=\varphi(\zeta)$ and $\varphi^{\prime}(\zeta) \neq \psi^{\prime}(\zeta)$; that is, $\varphi$ and $\psi$ must have the same first-order boundary data.

$d$-composition operators on the weighted Bergman space are highly connected with composition operators on weighted Dirichlet space. In the following, we will use the symbol $C$ to denote a finite positive number which is not necessarily the same at each occurrence.

Lemma 6. For $\alpha>-1$ and $1<p \leq q<\infty$, let $\varphi, \psi \in \mathscr{D}_{\alpha}^{p}$ be such that $\varphi(\mathbb{D}) \subset \mathbb{D}$ and $\psi(\mathbb{D}) \subset \mathbb{D}$. Then $C_{\varphi}-C_{\psi}$ is bounded (compact) from $\mathscr{D}_{\alpha}^{p}$ into $\mathscr{D}_{\alpha}^{q}$ if and only if $D_{\varphi}-D_{\psi}$ is bounded (compact) from $A_{\alpha}^{p}$ into $A_{\alpha}^{q}$.

Proof. We just verify the condition of boundedness. Suppose $C_{\varphi}-C_{\psi}$ is bounded from $\mathscr{D}_{\alpha}^{p}$ into $\mathscr{D}_{\alpha}^{q}$. Then there exists a constant $C>0$ such that

$$
\left\|\left(C_{\varphi}-C_{\psi}\right) f\right\|_{\mathscr{D}_{\alpha}^{q}} \leq C\|f\|_{\mathscr{D}_{\alpha}^{p}}
$$

for all $f \in \mathscr{D}_{\alpha}^{p}$. Take $f \in A_{\alpha}^{p}$ and let the function $g \in \mathscr{D}_{\alpha}^{p}$ be such that $g^{\prime}=f$ and $g(0)=0$. Then we have

$$
\begin{aligned}
\left\|\left(D_{\varphi}-D_{\psi}\right) f\right\|_{A_{\alpha}^{q}}^{q} & =\left\|\varphi^{\prime} f \circ \varphi-\psi^{\prime} f \circ \psi\right\|_{A_{\alpha}^{q}}^{q} \\
& =\left\|(g \circ \varphi-g \circ \psi)^{\prime}\right\|_{A_{\alpha}^{q}}^{q}
\end{aligned}
$$

$$
\begin{aligned}
& =\|g \circ \varphi-g \circ \psi\|_{D_{\alpha}^{q}}^{q} \\
& =\left\|\left(C_{\varphi}-C_{\psi}\right) g\right\|_{\mathscr{D}_{\alpha}^{q}}^{q} \leq C\|g\|_{\mathscr{D}_{\alpha}}^{q} \\
& =C\|f\|_{A_{\alpha}^{q}}^{q} .
\end{aligned}
$$

Thus $D_{\varphi}-D_{\psi}: A_{\alpha}^{p} \rightarrow A_{\alpha}^{q}$ is bounded.

Conversely, assume $D_{\varphi}-D_{\psi}: A_{\alpha}^{p} \rightarrow A_{\alpha}^{q}$ is bounded. Take $f \in \mathscr{D}_{\alpha}^{p}$ such that $f(0)=0$. Then we have

$$
\begin{aligned}
\left\|\left(C_{\varphi}-C_{\psi}\right) f\right\|_{D_{\alpha}}^{q} & =\left\|(f \circ \varphi-f \circ \psi)^{\prime}\right\|_{A_{\alpha}^{q}}^{q} \\
& =\left\|\left(D_{\varphi}-D_{\psi}\right)\left(f^{\prime}\right)\right\|_{A_{\alpha}^{q}}^{q} \leq C\left\|f^{\prime}\right\|_{A_{\alpha}^{p}}^{p} \\
& =C\|f\|_{D_{\alpha}}^{p} .
\end{aligned}
$$

Theorem 7. Suppose $\varphi$ is an analytic self-map of unit disk and has finite angular derivative $\varphi^{\prime}(\zeta)$ at some point $\zeta$ in the unit circle. Let $\psi$ be another analytic self-map of unit disk and consider $C_{\varphi}$ and $C_{\psi}$ acting on D. Then unless both

(1) $\varphi(\zeta)=\psi(\zeta)$,

(2) $\varphi^{\prime}(\zeta)=\psi^{\prime}(\zeta)$

we have $\left\|C_{\varphi}-C_{\psi}\right\|_{e}^{2} \geq C$, where the constant $C$ depends only on the spaces $\mathscr{D}$.

Proof. Note that $\mathscr{D}=\mathscr{D}_{0}^{2}$ and $A^{2}=A_{0}^{2}$. Setting $\alpha=0$ and $p=q=2$ in Lemma 6, from the proof of Lemma 6, we know there exist two constants $C_{1}$ and $C_{2}$ such that

$$
C_{1}\left\|D_{\varphi}-D_{\psi}\right\|_{A^{2}} \leq\left\|C_{\varphi}-C_{\psi}\right\|_{\mathscr{D}} \leq C_{2}\left\|D_{\varphi}-D_{\psi}\right\|_{A^{2}} .
$$

The desired result now follows from Theorem 2.

Corollary 8. If $\varphi$ has finite angular derivative on a set of positive measures, then $C_{\varphi}$ is isolated in $\mathscr{C}(\mathscr{D})$. For any $\psi \neq \varphi$, $\left\|C_{\varphi}-C_{\psi}\right\|_{e}^{2} \geq C$, where constant $C$ depends only on the $\mathscr{D}$.

Corollary 9. Suppose $C_{\psi}$ is in the component of $\mathscr{C}(\mathscr{D})$ containing $C_{\varphi}$. Then $\varphi$ and $\psi$ must have the same first-order boundary data, where $\varphi$ has finite angular derivative.

\section{Compact Composition Operators on Dirichlet Space}

The proof of the following lemma is routine, which is omitted here.

Lemma 10. Let $\varphi$ be an analytic self-map of $\mathbb{D}$ such that the induced composition operator $C_{\varphi}$ is bounded on $\mathscr{D}$. Then $C_{\varphi}$ acts on $\mathscr{D}$ compactly if and only if whenever $\left\{f_{n}\right\}$ is a bounded sequence in $\mathscr{D}$ converging to zero uniformly on compact subsets of $\mathbb{D}$, then $\left\|C_{\varphi}\left(f_{n}\right)\right\| \rightarrow 0$. 
It is well known (e.g., Proposition 9.9 in [1]) that the compact composition operators on $\mathscr{D}_{\alpha}$ form an arcwise connected set in $\mathscr{C}\left(\mathscr{D}_{\alpha}\right)$ for all $\alpha \geq 1$. By Lemma 10, we find the result is also true on the Dirichlet $\mathscr{D}=\mathscr{D}_{0}$ if we note that if $\|\varphi\|_{\infty}<1$, then $C_{\varphi}$ is compact on the Dirichlet space.

Theorem 11. The compact composition operators on $\mathscr{D}$ form an arcwise connected set in $\mathscr{C}(\mathscr{D})$.

The proof of this theorem is almost the same as the proof of Proposition 9.9 in [1], so we just give an outline here.

Proof. Given $C_{\varphi}$ and $C_{\psi}$ compact on $\mathscr{D}$ we first construct a continuous map of $[0,1]$ into $\mathscr{C}(\mathscr{D})$ taking 1 to $C_{\varphi}$ and 0 to $C_{\varphi_{0}}$, the composition operator whose symbol is the constant map $\varphi(0)$. The path $t \mapsto C_{\varphi_{t}}$, where $\varphi_{t}(z)=\varphi(t z)$, will satisfy our demand $\left(\left\|\varphi_{t}\right\|_{\infty}<1\right.$ implying the compactness of $\left.C_{\varphi_{t}}\right)$.

Secondly, we construct continuous arc joining.

$C_{\varphi_{0}}$ to $C_{\psi_{0}}$. Let $L(t)=(1-t) \varphi(0)+t \psi(0)$ be the line segment in $\mathbb{D}$ from $\varphi(0)$ to $\psi(0)$ and then map $[0,1]$ into $\mathscr{C}(\mathscr{D})$ by $t \mapsto C_{L(t)}$, where $C_{L(t)} f(z)=f(L(t))$. It is easy to verify that this map is continuous.

It follows from Lemma 6 and Theorem 11 that we have following corollary.

Corollary 12. The compact d-composition operators on $A^{2}(\mathbb{D})$ form an arcwise connected set in $\mathscr{C}_{d}\left(A^{2}\right)$.

From Theorem 11, we find that the compact composition operators on $\mathscr{D}$ are in the same component, but we do not know whether there is any noncompact composition operator belonging to the component generated by compact ones on $\mathscr{D}$. The answers to those similar questions on different analytic function spaces appear somewhat different. One has positive answers when the spaces are classical Bergman space, Bloch space, and $H^{\infty}$, while the answer is negative on classical Hardy space.

Remark 13. Let $\varphi \in S(\mathbb{D})$ be a linear-fractional self-map of the unit disk with $\varphi(\zeta)=\eta$, where both $\zeta$ and $\eta$ are unimodular. Then the induced composition operator $C_{\varphi}$ cannot be in the component generated by compact composition operators on Dirichlet space. Suppose $C_{\varphi}$ did. The linear-fractional self-map $\varphi$ must have finite angular derivative at $\zeta$. And the composition operator induced by $z / 2$ is compact on D. By Theorem 11 compact composition operators must be in the same component and $C_{\varphi}$ and $C_{z / 2}$ are in the same component. By Corollary $9 \varphi$ and $z / 2$ must satisfy SBD. That is impossible.

Lemma 14 (Corollary 2 in [5]). Let $u$ be an analytic function on $\mathbb{D}$ and let $\varphi$ be an analytic self-map of $\mathbb{D}$. Then the weighted composition operator $u C_{\varphi}$ is compact on $A^{2}$ if and only if $\lim _{|a| \rightarrow 1}\left\|\left(u C_{\varphi}\right) k_{a}\right\|^{2}=0$, where $k_{a}(z)$ is the normalized reproducing kernel of $A^{2}$ at a.

There is a practical corollary of Lemma 14.
Lemma 15 (Proposition 1 in [5]). Let $u$ be an analytic function on $\mathbb{D}$ and $\varphi \in S(\mathbb{D})$. If the weighted composition operator $u C_{\varphi}$ is compact on $A^{2}$, then

$$
\lim _{|z| \rightarrow 1} \frac{\left(1-|z|^{2}\right)|u(z)|}{1-|\varphi(z)|^{2}}=0 .
$$

From this lemma, in next section we will see that some linear-fractional composition operators cannot be compact on Dirichlet space, and we will discuss that component in $\mathscr{C}(\mathscr{D})$ through the characterization of the component in $\mathscr{C}_{d}\left(A^{2}\right)$.

\section{Linear-Fractional $d$-Composition Operators}

Throughout this section, $\varphi$ and $\psi$ will denote linear-fractional self-mappings of $\mathbb{D}$. To determine when linear-fractional $d$ composition operators belong to the same component of $\mathscr{C}_{d}\left(A^{2}\right)$, we need to state some lemmas.

Lemma 16 (Theorem 4.2.9 in [3]). Suppose $n \geq 1$ and $f$ is analytic in $\mathbb{D}$; then $f \in A^{2}(\mathbb{D})$ if and only if $\left(1-|z|^{2}\right)^{n} f^{(n)}(z)$ is in $A^{2}(\mathbb{D})$.

If $\varphi$ and $\psi$ are linear-fractional self-maps of the unit disk with the same boundary data, $\varphi(\zeta)=\psi(\zeta)=\eta$, where $\zeta$ and $\eta$ are in the unit circle. Then it is trivial that $D_{\varphi}$ and $D_{\psi}$ are in the same path component in $\mathscr{C}_{d}\left(A^{2}\right)$ if and only if $D_{\bar{\eta} \varphi(\zeta z)}$ and $D_{\bar{\eta} \psi(\zeta z)}$ are in the same path component in $\mathscr{C}_{d}\left(A^{2}\right)$.

So we can just discuss the situations when $\varphi(1)=1$ and $\varphi^{\prime}(1)=s \in \mathbb{R}$. The following lemma follows Lemma 3.2 in $[13]$ and its proof.

Lemma 17. Suppose that $\varphi$ is a nonautomorphic, linearfractional self-mapping of $\mathbb{D}$ such that $\varphi(1)=1$ and $\varphi^{\prime}(1)=s$. Denote $T(z)=(1+z) /(1-z)$ for $z \in \mathbb{D}$; then there is a complex constant a with positive real part such that

$$
\begin{aligned}
\varphi(z) & =T^{-1}\left(\frac{1}{s} T(z)+a\right) \\
& =\frac{(-1+a s-s) z-1-a s+s}{(s-1+a s) z-s-1-a s} .
\end{aligned}
$$

Conversely if we put $\varphi(z)=((-1+a s-s) z-1-a s+$ $s) /((s-1+a s) z-s-1-a s)$, it is clear that $\varphi$ is a linearfractional self-mapping of $\mathbb{D}$ such that $\varphi(1)=1$ and $\varphi^{\prime}(1)=s$. By Lemma 15 and direct computation, it is easy to check all $C_{\varphi}$ are noncompact on $\mathscr{D}$. So all $D_{\varphi}$ are also noncompact on $A^{2}$ equivalently by Lemma 6 . 
Lemma 18. Let $r:[0,1] \rightarrow \mathbb{C}$ be defined by $r(t)=a+t(b-a)$ so that $r$ is the straight-line path in the open right half-plane joining $a$ and $b$. Let $\varphi_{t}$ be the self-map of $\mathbb{D}$ given by

$$
\begin{aligned}
\varphi_{t}(z) & =T^{-1}\left(\frac{1}{s} T(z)+r(t)\right) \\
& =\frac{(-1+r(t) s-s) z-1-r(t) s+s}{(s-1+r(t) s) z-s-1-r(t) s} .
\end{aligned}
$$

Then $t \mapsto D_{\varphi_{t}}$ is continuous from the unit interval to $\mathscr{C}_{d}\left(A^{2}\right)$, placing both $D_{\varphi}$ and $D_{\psi}$ in the same path component.

Proof. Let $t_{1}$ and $t_{2}$ be distinct points in $[0,1]$ with $t_{1} \leq t_{2}$. This lemma will be proved if we proved that there is constant $M$ such that

$$
\left\|D_{\varphi_{t_{2}}}-D_{\varphi_{t_{1}}}\right\|^{2} \leq M\left(t_{2}-t_{1}\right)^{2} .
$$

We require some preliminary estimates. Fix $z$ in the closure of $\mathbb{D}$, let $\varphi_{z}(t)=\varphi_{t}(z)$, and calculate the derivative of $\varphi_{z}$ with respect to $t$ :

$$
\varphi_{z}^{\prime}(t)=\frac{2(b-a) s^{2}(1-z)^{2}}{((s-1+r(t) s) z-s-1-r(t) s)^{2}} .
$$

For any $z \in \overline{\mathbb{D}}$ and $t \in[0,1]$, note that $|r(t)| \leq|a|+|b|$; we have

$$
\begin{aligned}
& |(s-1+r(t) s) z|+|s+1+r(t) s| \\
& \quad \leq 2(s+1+(|a|+|b|) s), \\
& |(s-1+r(t) s) z-s-1-r(t) s| \\
& \quad \geq|s+1+r(t) s|-|s-1+r(t) s| .
\end{aligned}
$$

Since $\operatorname{Re}(r(t))>0$,

$$
\begin{aligned}
& |s+1+r(t) s|^{2}-|s-1+r(t) s|^{2}=4 s+4 \mathbf{R e}(r(t)) s \\
& \quad>4 s,
\end{aligned}
$$

it follows from (30) that

$$
\begin{aligned}
& |(s-1+r(t) s) z-s-1-r(t) s| \\
& \quad \geq \frac{|s+1+r(t) s|^{2}-|s-1+r(t) s|^{2}}{|(s-1+r(t) s) z|+|s+1+r(t) s|} \\
& \quad \geq \frac{4 s}{2(s+1+(|a|+|b|) s)}=\frac{2 s}{s+1+(|a|+|b|) s} .
\end{aligned}
$$

Combining (29) and (32), there is a constant $K_{1}=s|b-a|(s+$ $1+s(|a|+|b|))$ such that

$$
\left|\varphi_{z}^{\prime}(t)\right| \leq K_{1}|1-z|^{2}
$$

for all $z \in \overline{\mathbb{D}}$ and $t \in[0,1]$. Calculate the derivative of $\varphi_{t}$ with respect to $z$ :

$$
\varphi_{t}^{\prime}(z)=\frac{4 s}{((s-1+r(t)) z-s-1-r(t) s)^{2}} .
$$

Similarly we have

$$
\left|\varphi_{t}^{\prime}(z)\right| \leq K_{2}
$$

for a constant $K_{2}$ independent of $z \in \overline{\mathbb{D}}$ and $t \in[0,1]$. An easy calculation shows that

$$
\frac{1-z}{1-\varphi_{t}(z)}=\frac{s+1+r(t) s-(s-1+r(t) s) z}{2 s} .
$$

Thus, applying the triangle inequality, we see that, for every $z \in \mathbb{D}$

$$
\frac{|1-z|}{\left|1-\varphi_{t}(z)\right|} \leq \frac{s+1+(|a|+|b|) s}{s}=K_{3} .
$$

Because $\varphi_{t}(\mathbb{D})$ is a proper subdisk of $\mathbb{D}$ internally tangent to the unit circle at 1 , we may set

$$
\varphi_{t}(\mathbb{D})=\left\{w \in \mathbb{D}:\left|w-w_{0}\right|<R\right\},
$$

where $1-w_{0}=R$; that is, $w_{0}=1-R$. It is easy to show that

$$
\begin{aligned}
w & \in \varphi_{t}(\mathbb{D}) \Longleftrightarrow \\
|w-(1-R)|^{2} & <R^{2} \Longleftrightarrow \\
|1-w|^{2} & <\frac{R}{1-R}\left(1-|w|^{2}\right),
\end{aligned}
$$

and this implies that

$$
\frac{\left|1-\varphi_{t}(z)\right|^{2}}{1-\left|\varphi_{t}(z)\right|^{2}}<\frac{R}{1-R}
$$

for any $z \in \mathbb{D}$.

It follows from (27) that $T\left(\varphi_{t}(z)\right)=T(z) / s+r(t)$. And the image of the circle

$$
\frac{|1-w|^{2}}{1-|w|^{2}}=\frac{R}{1-R}
$$

under $T(z)=(1+z) /(1-z)$ is the line $\operatorname{Re}(r(t))+k i$, where $k \in \mathbb{R}$ in right half-plane, and we put $T^{-1}(\boldsymbol{R e}(r(t))+k i)$ back into (41). We have that

$$
\begin{aligned}
\frac{1-R}{R} & =\frac{\left|1-T^{-1}(\operatorname{Re}(r(t))+k i)\right|^{2}}{1-\left|T^{-1}(\operatorname{Re}(r(t))+k i)\right|^{2}} \\
& =\frac{4}{|\operatorname{Re}(r(t))+1+k i|^{2}-|\operatorname{Re}(r(t))-1+k i|^{2}} \\
& =\frac{1}{\operatorname{Re}(r(t))} .
\end{aligned}
$$

Consequently,

$$
\begin{aligned}
\frac{\left|1-\varphi_{t}(z)\right|^{2}}{1-\left|\varphi_{t}(z)\right|^{2}} & \leq(\operatorname{Re}(r(t)))^{-1} \\
& \leq(\min \{\operatorname{Re}(a), \operatorname{Re}(b)\})^{-1}=K_{4} .
\end{aligned}
$$


For any $f \in A^{2}$, we have that

$$
f\left(\varphi_{t_{2}}(z)\right)-f\left(\varphi_{t_{1}}(z)\right)=\int_{t_{1}}^{t_{2}} f^{\prime}\left(\varphi_{z}(t)\right) \varphi_{z}^{\prime}(t) d t
$$

This produces the crucial estimate:

$$
\begin{aligned}
& \left\|\left(D_{\varphi_{t_{2}}}-D_{\varphi_{t_{1}}}\right) f\right\|^{2}=\int_{\mathbb{D}} \mid \varphi_{t_{2}}^{\prime}(z) f\left(\varphi_{t_{2}}(z)\right)-\varphi_{t_{1}}^{\prime}(z) \\
& \left.\cdot f\left(\varphi_{t_{1}}(z)\right)\right|^{2} d A(z) \\
& =\int_{\mathbb{D}} \mid\left[\varphi_{t_{2}}^{\prime}(z)-\varphi_{t_{1}}^{\prime}(z)\right] f\left(\varphi_{t_{2}}(z)\right)+\varphi_{t_{1}}^{\prime}(z) \\
& \left.\cdot\left[f\left(\varphi_{t_{2}}(z)\right)-f\left(\varphi_{t_{1}}(z)\right)\right]\right|^{2} d A(z) \\
& \leq 2 \int_{\mathbb{D}}\left|1-\frac{\varphi_{t_{1}}^{\prime}(z)}{\varphi_{t_{2}}^{\prime}(z)}\right|^{2}\left|\varphi_{t_{2}}^{\prime}(z)\right|^{2} \\
& \cdot\left|f\left(\varphi_{t_{2}}(z)\right)\right|^{2} d A(z) \\
& +2 \int_{\mathbb{D}}\left|\varphi_{t_{1}}^{\prime}(z)\right|^{2} \mid f\left(\varphi_{t_{2}}(z)\right) \\
& -\left.f\left(\varphi_{t_{1}}(z)\right)\right|^{2} d A(z)=2\left(I_{1}+I_{2}\right), \\
& I_{1}=\int_{\mathbb{D}} \mid 1 \\
& -\left.\left(\frac{\left(s-1+r\left(t_{2}\right) s\right) z-s-1-r\left(t_{2}\right) s}{\left(s-1+r\left(t_{1}\right) s\right) z-s-1-r\left(t_{1}\right) s}\right)^{2}\right|^{2} \\
& \cdot\left|\varphi_{t_{2}}^{\prime}(z)\right|^{2}\left|f\left(\varphi_{t_{2}}(z)\right)\right|^{2} d A(z) \leq K_{5}^{4}\left(t_{2}\right. \\
& \left.-t_{1}\right)^{2} \int_{\mathbb{D}}\left|\varphi_{t_{2}}^{\prime}(z)\right|^{2}\left|f\left(\varphi_{t_{2}}(z)\right)\right|^{2} d A(z) \leq K_{5}^{4}\left(t_{2}\right. \\
& \left.-t_{1}\right)^{2}\|f\|^{2}
\end{aligned}
$$

where the constant $K_{5}$ denotes upper bound of $\mid(s-1+$ $r(t) s) z-s-1-\left.r(t) s\right|^{-1}$ multiplied by an absolute constant:

$$
\begin{aligned}
I_{2} & \leq K_{2} \int_{\mathbb{D}}\left|f\left(\varphi_{t_{2}}(z)\right)-f\left(\varphi_{t_{1}}(z)\right)\right|^{2} d A(z) \\
& =K_{2} \int_{\mathbb{D}}\left|\int_{t_{1}}^{t_{2}} f^{\prime}\left(\varphi_{z}(t)\right) \varphi_{z}^{\prime}(t) d t\right|^{2} d A(z) \\
& \leq K_{2}\left(t_{2}-t_{1}\right) \int_{\mathbb{D}} \int_{t_{1}}^{t_{2}}\left|f^{\prime}\left(\varphi_{z}(t)\right) \varphi_{z}^{\prime}(t)\right|^{2} d t d A(z) \\
& \leq 4|b-a|^{2} s^{4} K_{2}\left(t_{2}-t_{1}\right) \int_{t_{1}}^{t_{2}} \int_{\mathbb{D}}\left|f^{\prime}\left(\varphi_{t}(z)\right)\right|^{2}
\end{aligned}
$$

$$
\begin{aligned}
& \cdot\left|\varphi_{t}^{\prime}(z)\right|^{2}|1-z|^{4} d A(z) d t \leq K_{3}^{4} K_{4}^{2} K_{6}\left(t_{2}\right. \\
& \left.-t_{1}\right) \int_{t_{1}}^{t_{2}} \int_{\mathbb{D}}\left|f^{\prime}\left(\varphi_{t}(z)\right)\right|^{2}\left|\varphi_{t}^{\prime}(z)\right|^{2} \\
& \cdot\left(1-\left|\varphi_{t}(z)\right|^{2}\right)^{2} d A(z) d t \leq K_{3}^{4} K_{4}^{2} K_{6}\left(t_{2}\right. \\
& \left.-t_{1}\right) \int_{t_{1}}^{t_{2}} d t \int_{\mathbb{D}}\left|f^{\prime}(w)\right|^{2}\left(1-|w|^{2}\right)^{2} d A(w) \\
& =K_{3}^{4} K_{4}^{2} K_{6}\left(t_{2}-t_{1}\right)^{2}\left\|\left(1-|w|^{2}\right) f^{\prime}(w)\right\|^{2} \\
& \leq K_{3}^{4} K_{4}^{2} K_{6}\left(t_{2}-t_{1}\right)^{2} C\|f\|^{2},
\end{aligned}
$$

where $K_{6}=4|b-a|^{2} s^{4} K_{2}$ and last inequality follows by using Lemma 16. Thus we have

$$
\begin{aligned}
& \left\|\left(D_{\varphi_{t_{2}}}-D_{\varphi_{t_{1}}}\right) f\right\|^{2} \\
& \quad \leq 2\left(K_{5}^{4}+K_{3}^{4} K_{4}^{2} K_{6} C\right)\|f\|^{2}\left(t_{2}-t_{1}\right)^{2},
\end{aligned}
$$

where $K_{j}(j=3,4,5,6)$ and $C$ depend only on $a, b, s$. This completes the proof of the lemma.

Theorem 19. Suppose that $\varphi$ and $\psi$ are linear-fractional selfmappings of $\mathbb{D}$. The following are equivalent:

(a) $D_{\varphi}$ and $D_{\psi}$ lie in the same component of $\mathscr{C}_{d}\left(A^{2}\right)$.

(b) $\varphi$ and $\psi$ satisfy $S B D$.

(c) $D_{\varphi}$ and $D_{\psi}$ are joined by a continuous path in $\mathscr{C}_{d}\left(A^{2}\right)$.

Proof. Clearly (c) implies (a). That (a) implies (b) is proved in Theorem 5. Thus to complete the proof of Theorem 19, we establish (b) implies (c).

If $\varphi$ is an automorphism, then, for any $\zeta$ in the unit circle, $|\varphi(\zeta)|=1$. That $\varphi$ and $\psi$ satisfy SBD implies $\psi(\zeta)=\varphi(\zeta)$ for any $\zeta$ in the unit circle. It turns out that $\psi=\varphi$ by Maximum Principle, and the conclusion is obvious.

Suppose that $\varphi$ is not an automorphism; then $\varphi(\mathbb{D})$ is a proper subdisk of $\mathbb{D}$; there are exactly two possibilities:

(i) $|\varphi(\zeta)|<1$ for any $\zeta \in \partial \mathbb{D}$.

(ii) There exists a point $\zeta \in \partial \mathbb{D}$ such that $|\varphi(\zeta)|=1$.

Suppose that (i) applies and $\varphi$ and $\psi$ satisfy SBD. Then $\psi$ also must map the closure of $\mathbb{D}$ into $\mathbb{D}$. In this situation, both $C_{\varphi}$ and $C_{\psi}$ are compact operators on $\mathscr{D}$. By Lemma 17, we have both $D_{\varphi}$ and $D_{\psi}$ are compact on $A^{2}(\mathbb{D})$. Compact $d$-composition operators are shown to belong to the same arcwise connected subset in Corollary 12. Thus, to complete the proof of the theorem, we need to handle case (ii).

We may suppose that $\varphi(1)=1$. Our setting condition is that $\psi$ share the same first-order boundary data with $\varphi$ so $\psi(1)=1$ and we denote $s=\varphi^{\prime}(1)=\psi^{\prime}(1)$. By Lemma 17, 
there are two constants $a$ and $b$ satisfying $\operatorname{Re}(a)>0$ and $\boldsymbol{R e}(b)>0$, such that

$$
\begin{aligned}
& T(\varphi(z))=\frac{1}{s} T(z)+a, \\
& T(\psi(z))=\frac{1}{s} T(z)+b .
\end{aligned}
$$

Denote $\varphi_{t}$ described by Lemma 18; then $t \mapsto D_{\varphi_{t}}$ is continuous from the unit interval to $\mathscr{C}_{d}\left(A^{2}\right)$, placing both $D_{\varphi}$ and $D_{\psi}$ in the same path component by Lemma 18. This ends the proof of the theorem.

Next corollary is an obvious consequence of Theorem 19 and gives a partial description of component in $\mathscr{C}(\mathscr{D})$.

Corollary 20. Suppose that $\varphi$ and $\psi$ are linear-fractional selfmappings of $\mathbb{D}$. The following are equivalent:

(a) $C_{\varphi}$ and $C_{\psi}$ lie in the same component of $\mathscr{C}(\mathscr{D})$.

(b) $\varphi$ and $\psi$ satisfy SBD.

(c) $C_{\varphi}$ and $C_{\psi}$ are joined by a continuous path in $\mathscr{C}(\mathscr{D})$.

\section{Conflict of Interests}

The authors declare that there is no conflict of interests regarding the publication of this paper.

\section{Acknowledgments}

The work was supported in part by National Science Foundation of China (Grant nos. 11301132; 11371276; 11501415; and 11171087) and Natural Science Foundation of Hebei Province (Grant no. A2013202265) and Tianjin Advanced Education Development Fund (Grant no. 20111005).

\section{References}

[1] C. C. Cowen and B. D. MacCluer, Composition Operators on Spaces of Analytic Functions, Studies in Advanced Mathematics, CRC Press, Boca Raton, Fla, USA, 1995.

[2] J. H. Shapiro, Composition Operators and Classical Function Theory, Universitext: Tracts in Mathematics, Springer, New York, NY, USA, 1993.

[3] K. H. Zhu, Operator Theory in Function Spaces, Marcel Dekker, New York, NY, USA, 1990.

[4] K. H. Zhu, Spaces of Holomorphic Functions in the Unit Ball, Graduate Texts in Mathematics, Springer, New York, NY, USA, 2005.

[5] Ž. Čučkovič and R. Zhao, "Weighted composition operators on the Bergman space," Journal of the London Mathematical Society, vol. 70, no. 2, pp. 499-511, 2004.

[6] C. Hammond and B. D. MacCluer, "Isolation and component structure in spaces of composition operators," Integral Equations and Operator Theory, vol. 53, no. 2, pp. 269-285, 2005.

[7] Z. H. Zhou and R. Y. Chen, "Weighted composition operators fom $F(p, q, s)$ to Bloch type spaces," International Journal of Mathematics, vol. 19, no. 8, pp. 899-926, 2008.
[8] Z. H. Zhou and J. H. Shi, "Compactness of composition operators on the Bloch space in classical bounded symmetric domains," The Michigan Mathematical Journal, vol. 50, no. 2, pp. 381-405, 2002.

[9] E. Berkson, "Composition operators isolated in the uniform operator topology," Proceedings of the American Mathematical Society, vol. 81, no. 2, pp. 230-232, 1981.

[10] B. D. MacCluer, "Components in the space of composition operators," Integral Equations and Operator Theory, vol. 12, no. 5, pp. 725-738, 1989.

[11] J. H. Shapiro and C. Sundberg, "Isolation amongst the composition operators," Pacific Journal of Mathematics, vol. 145, no. 1, pp. 117-152, 1990.

[12] E. A. Gallardo-Gutiérrez, M. J. González, P. J. Nieminen, and E. Saksman, "On the connected component of compact composition operators on the Hardy space," Advances in Mathematics, vol. 219, no. 3, pp. 986-1001, 2008.

[13] P. S. Bourdon, "Components of linear-fractional composition operators," Journal of Mathematical Analysis and Applications, vol. 279, no. 1, pp. 228-245, 2003.

[14] J. Moorhouse, "Compact differences of composition operators," Journal of Functional Analysis, vol. 219, no. 1, pp. 70-92, 2005.

[15] T. Kriete and J. Moorhouse, "Linear relations in the Calkin algebra for composition operators," Transactions of the American Mathematical Society, vol. 359, no. 6, pp. 2915-2944, 2007.

[16] E. Saukko, "Difference of composition operators between standard weighted Bergman spaces," Journal of Mathematical Analysis and Applications, vol. 381, no. 2, pp. 789-798, 2011.

[17] E. Saukko, "An application of atomic decomposition in Bergman spaces to the study of differences of composition operators," Journal of Functional Analysis, vol. 262, no. 9, pp. 3872-3890, 2012.

[18] B. R. Choe, H. Koo, and I. Park, "Compact differences of composition operators over polydisks," Integral Equations and Operator Theory, vol. 73, no. 1, pp. 57-91, 2012.

[19] B. R. Choe, H. Koo, and I. Park, "Compact differences of composition operators on the Bergman spaces over the ball," Potential Analysis, vol. 40, no. 1, pp. 81-102, 2014.

[20] Z.-S. Fang and Z.-H. Zhou, "Differences of composition operators on the space of bounded analytic functions in the polydisc," Abstract and Applied Analysis, vol. 2008, Article ID 983132, 10 pages, 2008.

[21] Z.-S. Fang and Z.-H. Zhou, "Differences of composition operators on the Bloch space in the polydisc," Bulletin of the Australian Mathematical Society, vol. 79, no. 3, pp. 465-471, 2009.

[22] R. F. Allen, K. C. Heller, and M. A. Pons, "Compact differences of composition operators on weighted Dirichlet spaces," Central European Journal of Mathematics, vol. 12, no. 7, pp. 1040-1051, 2014. 


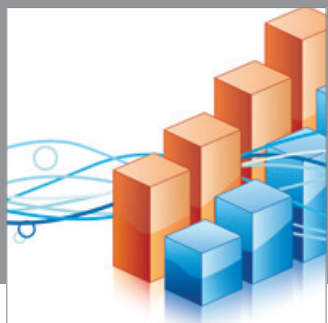

Advances in

Operations Research

mansans

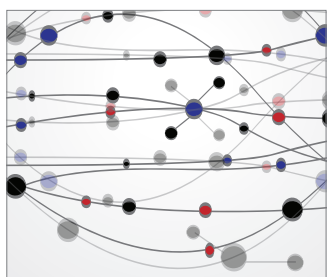

The Scientific World Journal
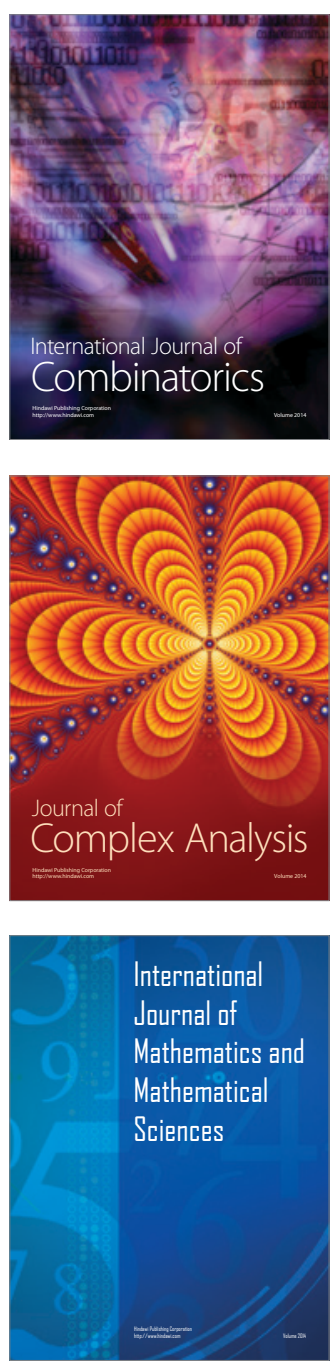
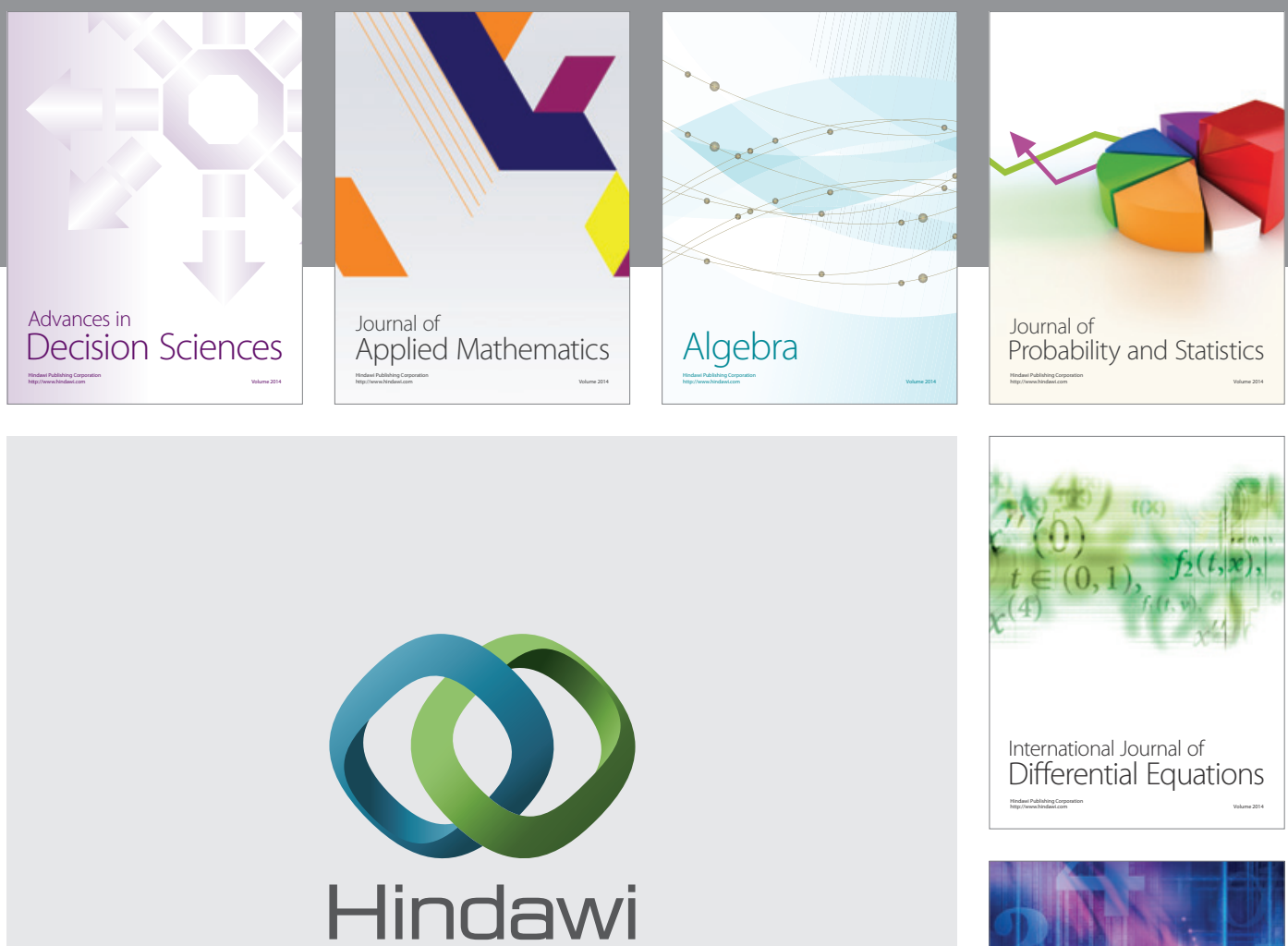

Submit your manuscripts at http://www.hindawi.com
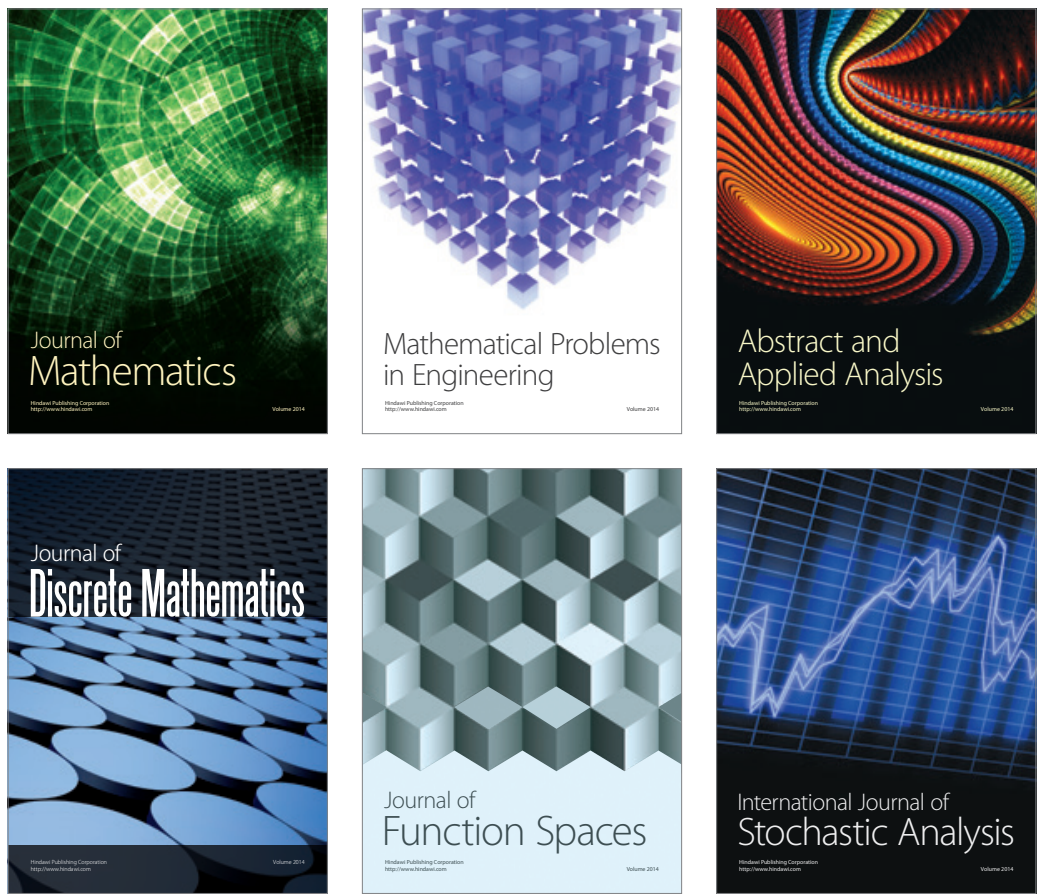

Journal of

Function Spaces

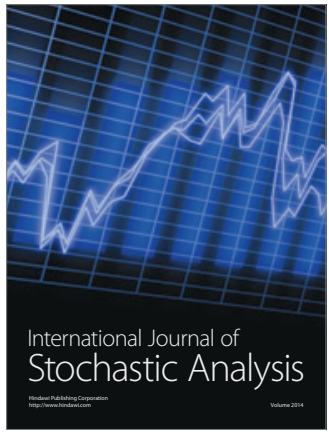

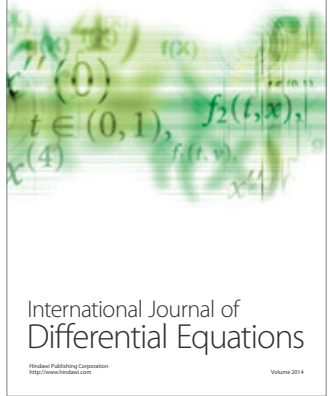
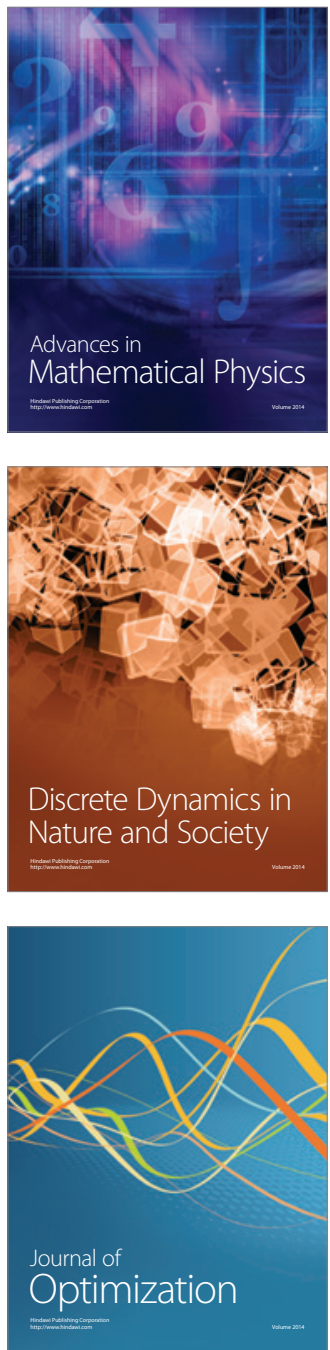